\title{
Article
}

\section{Indoor High-Precision 3D Positioning System Based on Visible-Light Communication Using Improved Whale Optimization Algorithm}

\author{
Xianmeng Meng ${ }^{1, *}$, Chaochuan Jia ${ }^{2}$, Cuicui Cai ${ }^{2}$, Fugui He ${ }^{2}$ and Qing Wang ${ }^{1}$ \\ 1 College of Electronic Engineering, Anhui Xinhua University, Hefei 230088, China; wangqing@axhu.edu.cn \\ 2 College of Electronics and Information Engineering, West Anhui University, Lu'an 237012, China; \\ ccjia@hfcas.ac.cn (C.J.); caicuihappy@wxc.edu.cn (C.C.); fuguihe@wxc.edu.cn (F.H.) \\ * Correspondence: mengxianmeng@axhu.edu.cn; Tel.: +86-18063062585
}

check for updates

Citation: Meng, X.; Jia, C.; Cai, C.; He, F.; Wang, Q. Indoor

High-Precision 3D Positioning System Based on Visible-Light Communication Using Improved Whale Optimization Algorithm. Photonics 2022, 9, 93. https:// doi.org/10.3390/photonics9020093

Received: 27 December 2021 Accepted: 4 February 2022

Published: 6 February 2022

Publisher's Note: MDPI stays neutral with regard to jurisdictional claims in published maps and institutional affiliations.

Copyright: (c) 2022 by the authors. Licensee MDPI, Basel, Switzerland. This article is an open access article distributed under the terms and conditions of the Creative Commons Attribution (CC BY) license (https:// creativecommons.org/licenses/by/ $4.0 /)$.

\begin{abstract}
Visible-light communication (VLC) is a promising method for indoor positioning. The received signal strength algorithm is the most widely used localization algorithm in visible-light positioning (VLP) systems. However, in a VLP system, the photodiode (PD) will have a small rotation angle during movement, which will result in a massive positioning error ignoring the angle. In this study, a three-dimensional (3D) indoor VLP system using an improved whale optimization algorithm (IWOA) is proposed to reduce the error caused by the PD rotation. Firstly, the model of the VLC system with the PD rotation angles is introduced. Secondly, a novel IWOA with an elite opposition-based learning strategy and Lévy flight strategy is proposed. The convergence speed and accuracy of the WOA are improved. Lastly, the IWOA algorithm is efficiently utilized to address the problem with the PD rotation in the indoor VLP system. Simulation results show that the average error of 3D positioning is $2.14 \mathrm{~cm}$ with no PD rotation. When the PD has a rotation angle, the average positioning error estimated by ignoring the rotation angle is $27.14 \mathrm{~cm}$, while that estimated by considering the rotation angle is $7.85 \mathrm{~cm}$. In the VLP system, the positioning error with the PD rotation angle is effectively reduced by the proposed algorithm, which can be applied in a variety of indoor location scenes.
\end{abstract}

Keywords: indoor 3D positioning; visible-light communication; improved whale optimization algorithm; positioning accuracy; received signal strength; rotation angle

\section{Introduction}

With the rapid development of wireless sensor networks and mobile services, locationbased services (LBSs) have been significantly expanded in recent years [1]. High-precision indoor positioning is gaining attention for its wide applications, including in museums, shopping malls, underground parking areas, and tunnels. Many technologies are applied to indoor positioning, with typical systems based on a global positioning system (GPS), radiofrequency identification (RFID), wireless local area network (WLAN), ultrawide-band radio (UWB), and visible-light communication (VLC). The GPSs are extensively used for outdoor positioning and have high location accuracy. When applied in the indoor environment, a GPS suffers from considerable signal attenuation due to the fading of satellite signal strength and shadow effect. Most WLAN positioning systems use the method of fingerprint matching, but it is difficult to create fingerprint libraries, and a WLAN is highly reliant on the environment. Positioning systems such as RFID and UWB have high positioning accuracy, but require additional hardware devices. VLC is an emerging communication technology which uses light-emitting diode (LED) emitters for high-speed data transmission. VLC technology is currently used in a variety of applications, such as light fidelity (Li-Fi) [2] and visible-light positioning (VLP). However, a positioning 
system based on VLC is becoming a research hotspot due to its high precision, low cost, good security, and anti-electromagnetic interference capability [3].

A VLC-based positioning system employs the signals to measure the position of a mobile device, and the positioning can be classified into two types based on the receiving terminal: image-sensor-based [4,5] and photodiode (PD)-based [6]. LEDs provide their ID or location information to image-sensor-based positioning systems, and the image sensor receives the signals and uses image information processing to estimate its position. An image-sensor-based positioning system facilitates high-precision positioning, but the image processing is time-consuming and complicated. The PD-based positioning system offers low cost and provides a wide range of indoor positioning applications.

Various schemes have been proposed for PD-based positioning systems, where the angle or distance between the transmitter and receiver is calculated through triangulation, such as time of arrival (TOA), time difference of arrival (TDOA), angle of arrival (AOA), and received signal strength (RSS) $[7,8]$. Owing to the high light-transmission rates and the short distances between LEDs and PDs, small time errors can result in large errors in the estimation of distances. Therefore, TOA and TDOA technologies need to adopt a strict time synchronization mechanism. Although the AOA technique does not require a strict time synchronization mechanism, a sensor array is used to receive radio signals, and the location system is very complicated and expensive. On the contrary, a positioning system based on RSS can easily estimate the distance from the received signal strength and obtain high-precision positioning. Hence, several visible-light positioning systems based on RSS have been presented in recent years [9-11].

It is well known that the use of metaheuristic algorithms comprises an effective technique for solving nonlinear optimization problems. Many metaheuristic algorithms have been applied to resolve complex engineering problems [12-15]. However, threedimensional (3D) indoor location systems based on visible-light communication can be viewed as a global optimization problem, and a variety of metaheuristic optimization algorithms have been used to address the problem. Cai et al. developed an indoor VLP system using particle swarm optimization, and an average distance error of $3.9 \mathrm{~mm}$ was obtained within 20 iterations in an indoor environment measuring $3 \mathrm{~m} \times 3 \mathrm{~m} \times 4 \mathrm{~m}$ [16]. Guan et al. proposed a VLP system, which used a modified genetic algorithm (GA) and artificial neural network, and an average error of positioning of $1.02 \mathrm{~cm}$ was achieved [17]. Huang et al. proposed an indoor VLP system based on a modified artificial fish swarm algorithm (MAFSA), and a positioning error of $4.05 \mathrm{~mm}$ was achieved in an indoor space of $4 \mathrm{~m} \times 4 \mathrm{~m} \times 6 \mathrm{~m}$ [18]. Xu et al. presented an indoor 3D visible-light location system using adaptive parameter particle swarm optimization, and the system achieved similar positioning results with a smaller swarm [19]. Chen et al. proposed an indoor VLP system based on an improved hybrid bat algorithm, and the system achieved an average positioning error of $1.16 \mathrm{~cm}$ in a space of $5 \mathrm{~m} \times 5 \mathrm{~m} \times 3 \mathrm{~m}$ [20]. Chen et al. proposed an indoor 3D VLC system based on an improved immune particle swarm optimization algorithm, which considered the influence of environmental interference, and an average positioning error of $3.12 \mathrm{~cm}$ was achieved [21].

The aforementioned positioning algorithms assumed that the LED and PD remain parallel when the PD is moving, and the positioning algorithms can achieve high-precision positioning. In an indoor positioning system, it is difficult to keep the PD and LED parallel during movement, resulting in random rotation angles [22]. A large positioning error would be observed if the LED and PD are assumed to be parallel. To solve the PD random-rotation problem, this paper proposes a 3D indoor location system based on visible-light communication using an improved whale optimization algorithm (IWOA). The final results show that the IWOA with elite opposition-based learning and Lévy flight strategies improves solution accuracy and convergence speed.

The rest of this paper is organized as follows: Section 2 describes the fundamentals of a VLC system model. The IWOA is proposed in Section 3. Simulation results are presented and analyzed in Section 4. Conclusions are discussed in Section 5. 


\section{VLC System Model}

The channel model of VLC and the indoor positioning system model are shown in Figure 1. Owing to the long distance between the LEDs and the PDs, the LEDs can be viewed as a Lambertian radiation source. According to the Lambertian radiation model, the channel current gain between the LEDs to PDs can be given by Equation (1) [23].

$$
H(0)_{L O S}=\left\{\begin{array}{ll}
\frac{(m+1) A}{2 \pi d^{2}} \cos ^{m}(\phi) T_{S}(\psi) g(\psi) \cos (\psi), & 0 \leq \psi \leq \psi_{c} \\
0, & \psi>\psi_{c}
\end{array},\right.
$$

where $A$ represents the effective PD area, $d$ is the distance between the PD and LED, and $\phi$ and $\psi$ denote the angle irradiance and angle of incidence, respectively. $\psi_{c}$ is the field of view of the receiver, $T_{S}(\psi)$ is the gain of the optical filter, and $m$ is the Lambertian order, defined as

$$
m=-\log 2 / \log \left(\cos \left(\phi_{1 / 2}\right)\right),
$$

where $\phi_{1 / 2}$ is the half-power angle of the LED. The gain of the optical concentrator $g(\psi)$ can be expressed as

$$
g(\psi)=\left\{\begin{array}{ll}
\frac{n^{2}}{\sin ^{2}\left(\psi_{c}\right)}, & 0 \leq \psi \leq \psi_{c} \\
0, & \psi>\psi_{c}
\end{array},\right.
$$

where $n$ represents the optical concentrator's refractive index. The receiving power of the PD $P_{r}$ can be expressed as

$$
P_{r}=P_{t} \times H(0)_{L O S}
$$

where $P_{t}$ is the emitting power of the LED.

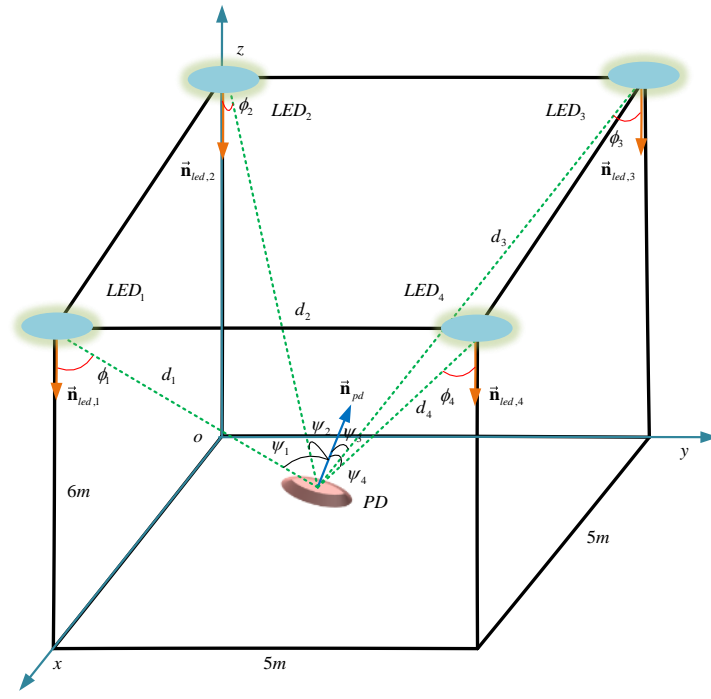

(a)

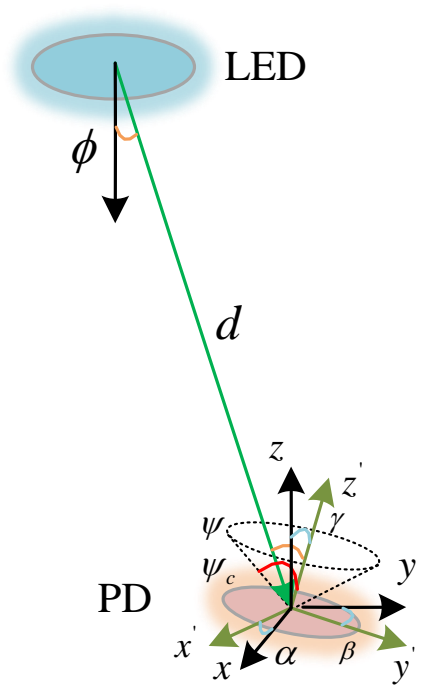

(b)

Figure 1. Diagram of (a) indoor positioning system and (b) the visible-light transmission channel model.

The VLC system mainly consists of shot noise $\sigma_{\text {shot }}^{2}$ and thermal noise $\sigma_{\text {thermal }}^{2}$, and the total noise $\sigma_{\text {noise }}^{2}$ is composed of both. The total noise is usually modeled as an additive white Gaussian noise (AWGN) [24]. The received signal strength can be expressed by the signal-to-noise ratio (SNR), represented as follows [25]:

$$
S N R=10 \log _{10} \frac{\left(P_{r} R_{p}\right)^{2}}{\sigma_{\text {shot }}^{2}+\sigma_{\text {thermal }}^{2}},
$$

where $R_{p}$ is the PD's optical conversion efficiency. 
Four LEDs are mounted on the ceiling board in a room, each of which is used for communication and illumination. A PD receives the signals from the four LEDs as a receiver, which is positioned at a random location in the room. Assuming that the normal of the $i$-th LED is $\overrightarrow{\mathbf{n}}_{l e d, i}=\left[\begin{array}{ll}0,0,-1\end{array}\right]^{T}$, the normal of the PD is $\overrightarrow{\mathbf{n}}_{p d}=\left[\begin{array}{lll}0 & 0 & 1\end{array}\right]^{T}$, and the vector from the $i$-th LED to PD is $\overrightarrow{\mathbf{d}}_{i}$, then $\cos \left(\phi_{i}\right)$ and $\cos \left(\psi_{i}\right)$ can be calculated as

$$
\begin{gathered}
\cos \left(\phi_{i}\right)=\frac{\overrightarrow{\mathbf{n}}_{l e d, i} \cdot \overrightarrow{\mathbf{d}}_{i}}{\left\|\overrightarrow{\mathbf{d}}_{i}\right\|_{\vec{n}}}=\frac{\overrightarrow{\mathbf{n}}_{l e d, i} \cdot \overrightarrow{\mathbf{d}}_{i}}{d_{i}} \\
\cos \left(\psi_{i}\right)=\frac{\overrightarrow{\mathbf{n}}_{p d} \cdot\left(-\overrightarrow{\mathbf{d}}_{i}\right)}{\left\|-\overrightarrow{\mathbf{d}}_{i}\right\|}=-\frac{\overrightarrow{\mathbf{n}}_{p d} \cdot \overrightarrow{\mathbf{d}}_{i}}{d_{i}}
\end{gathered}
$$

The RSS $P_{r}^{i}$ from the $i$-th LED is calculated as follows:

$$
\begin{aligned}
P_{r}^{i} & =\frac{-P_{t}^{i} \frac{(m+1) A}{2 \pi} T_{s}\left(\psi_{i}\right) g\left(\psi_{i}\right)}{d_{i}^{2}} \cos ^{m}\left(\phi_{i}\right) \cos \left(\psi_{i}\right) \\
= & \frac{-P_{t}^{i} \frac{(m+1) A}{2 \pi} T_{s}\left(\psi_{i}\right) g\left(\psi_{i}\right)}{d_{i}{ }^{m+3}}\left(\overrightarrow{\mathbf{n}}_{l e d, i} \cdot \overrightarrow{\mathbf{d}}_{i}\right)^{m}\left(\overrightarrow{\mathbf{n}}_{p d} \cdot \overrightarrow{\mathbf{d}}_{i}\right)
\end{aligned}
$$

At present, most of the positioning methods researched assume that the horizontal normal of the LEDs and the PD align with each other, and the angles of departure and arrival are equal. However, in a visible positioning system, it is difficult to maintain the parallel position while moving the PD. If the PD and LED are assumed to be parallel, a large positioning error will be observed. The PD has a small angle tilt, which can be considered a consequence of the PD rotation. Supposing that $\alpha, \beta$, and $\gamma$ represent the rotation angles of $P D$ about the $X$-axis, $Y$-axis, and $Z$-axis, respectively, the rotation matrix can then be defined as follows [26]:

$$
\begin{gathered}
R(\alpha, \beta, \gamma)=\operatorname{Rot}_{-} x(\alpha) * \text { Rot_y}_{-}(\beta) * \text { Rot_z}_{-}(\gamma) \\
=\left[\begin{array}{ccc}
1 & 0 & 0 \\
0 & \cos (\alpha) & \sin (\alpha) \\
0 & -\sin (\alpha) & \cos (\alpha)
\end{array}\right] \cdot\left[\begin{array}{ccc}
\cos (\beta) & 0 & -\sin (\beta) \\
0 & 1 & 0 \\
-\sin (\beta) & 0 & \cos (\beta)
\end{array}\right] \cdot\left[\begin{array}{ccc}
\cos (\gamma) & \sin (\gamma) & 0 \\
-\sin (\gamma) & \cos (\gamma) & 0 \\
0 & 0 & 1
\end{array}\right]
\end{gathered}
$$

Therefore, the normal vector $\overrightarrow{\mathbf{n}}_{p d}$ of the PD is changed as follows:

$$
\overrightarrow{\mathbf{n}}_{p d}^{\prime}=R(\alpha, \beta, \gamma) * \overrightarrow{\mathbf{n}}_{p d}
$$

The RSS $P_{r_{-} t i l t}^{i}$ from the $i$-th LED is then changed as follows:

$$
\begin{aligned}
P_{r_{-} t i l t}^{i} & =\frac{-P_{t}^{i} \frac{(m+1) A}{2 \pi} T_{S}\left(\psi_{i}\right) g\left(\psi_{i}\right)}{d_{i}{ }^{2}} \cos ^{m}\left(\phi_{i}\right) \cos \left(\psi_{i}\right) \\
= & \frac{-P_{t}^{i} \frac{(m+1) A}{2 \pi} T_{s}\left(\psi_{i}\right) g\left(\psi_{i}\right)}{d_{i}{ }^{m+3}}\left(\overrightarrow{\mathbf{n}}_{l e d, i} \cdot \overrightarrow{\mathbf{d}}_{i}\right)^{m}\left(\overrightarrow{\mathbf{n}}_{p d}^{\prime} \cdot \overrightarrow{\mathbf{d}}_{i}\right)
\end{aligned}
$$

Thus, a fitness function of visible-light location based on the RSS can be given by

$$
\text { Fitness }(\alpha, \beta, \gamma, x, y, z)=\sum_{i=1}^{4}\left(P_{r_{-} \text {meas }}^{i}-P_{r_{-} t i l t}^{i}\right)^{2}
$$

where $P_{r_{-} \text {meas }}^{i}$ represents the value of RSS measurement for the $i$-th LED, and $P_{r_{-}}^{i}$ tilt represents the value of RSS calculated by Equation (10). The optimal rotation angles and position of PD can be obtained by minimizing the fitness function. 


\section{Improved Whale Optimization Algorithm}

\subsection{Whale Optimization Algorithm}

The 3D indoor location problem can be solved with a global optimization algorithm, and the WOA is a novel metaheuristic algorithm for addressing the global optimization problem by mimicking the social behavior of humpback whales, as presented by Mirjalili and Lewis in 2016 [27]. The WOA is characterized by good optimization search ability, few controlling parameters, and simplicity of implementation.

The WOA uses three stages to update the position of individuals; these stages are surrounding prey, searching for prey, and spiral updating position [27].

Surrounding prey: If $p<0.5$ and $\left|A_{1}\right|<1$,

$$
\begin{gathered}
X_{i, G+1}=X_{\text {best }}-A_{1} \cdot D, \quad 0 \leq i \leq N P, \\
D=\left|C \cdot X_{b e s t}-X_{i, G}\right|, \\
A_{1}=2 \cdot a \cdot r-a, \\
C=2 \cdot r, \\
a=2-t \cdot\left(1-t / T_{\max }\right) .
\end{gathered}
$$

Search for prey: If $p<0.5$ and $\left|A_{1}\right| \geq 1$,

$$
\begin{gathered}
X_{i, G+1}=X_{\text {rand }, G}-A_{1} \cdot D, \\
D=\left|C \cdot X_{\text {rand }, G}-X_{i, G}\right| .
\end{gathered}
$$

Spiral updating position: If $p \geq 0.5$

$$
\begin{gathered}
X_{i, G+1}=X_{i, G}+D_{p} \cdot e^{b l} \cdot \cos (2 \pi l), \\
D_{p}=\left|X_{\text {best }}-X_{i, G}\right|,
\end{gathered}
$$

where $X_{i, G}$ is the solution of the current generation, $X_{i, G+1}$ is the solution of the next generation, $X_{\text {best }}$ is the best solution obtained thus far, $X_{\text {rand }, G}$ is a random solution chosen from the current generation, $p$ is a random number, $N P$ is the number of individuals in the population, $t$ is the current number of iterations, $T_{\max }$ is the maximum number of iterations, $a$ is linearly decreased from 2 to 0 over the course of multiple iterations, $r$ is a random number in the range $[0,1], b$ is a constant which defines the shape of the logarithmic spiral, and $l$ is a random real number in the range $[-1,1]$.

\subsection{Improved Whale Optimization Algorithm}

Similar to other metaheuristic algorithms, the WOA is predisposed to low convergence accuracy and premature convergence when dealing with complex optimization problems. The random initialization affects the performance of the algorithm, and the population diversity causes the algorithm to be trapped in a local optimum [28-30]. Therefore, to improve the search performance of the WOA, the IWOA with two strategies is proposed here.

\subsubsection{Elite Opposition-Based Learning}

Elite opposition-based learning (EOBL) is an innovative approach in the field of computational intelligence, and it has been applied in many metaheuristic algorithms [31-33]. An EOBL strategy is mainly used to improve the diversity of population by evaluating the fitness function of the current and opposition solutions, and then the better solutions are selected as the initial individual. The individual with the minimum value of fitness function is regarded as the elite individual in the population. The EOBL strategy can be expressed as follows [32]:

$$
\bar{X}_{i}=k(L+U)-X_{i}
$$




$$
X_{i}=\left\{\begin{array}{l}
\bar{X}_{i}, \text { Fitness }\left(\bar{X}_{i}\right)<\text { Fitness }\left(X_{i}\right) \\
X_{i}, \text { Fitness }\left(\bar{X}_{i}\right) \geq \text { Fitness }\left(X_{i}\right)
\end{array},\right.
$$

where $X_{i}$ and $\bar{X}_{i}$ are the current and opposition solutions, respectively, $k$ is a random number in the range $[0,1], L$ represents the lower boundaries of the scheduled area, and $U$ represents the upper boundaries of the scheduled area.

\subsubsection{Lévy Flight}

Lévy flight is a random search method that has been successfully used in a variety of metaheuristic algorithms [34-36]. Comparing the fitness values before and after the Lévy flight, the better solutions are selected to participate in next-generation evolution with a greedy strategy. The Lévy flight strategy can be expressed as

$$
\begin{gathered}
X_{i, G 1}=X_{i, G}+\operatorname{Levy}(\lambda) \times\left(X_{i, G}-X_{\text {best }}\right), \\
\operatorname{Levy}(\lambda) \sim|s|^{-1-\beta}, 1<\beta \leq 2, \\
s \sim \frac{\phi \times \mu}{|v|^{1 / \beta},} \\
\phi=\left(\frac{\Gamma(1+\beta) \times \sin \left(\frac{\pi \times \beta}{2}\right)}{\Gamma\left(\frac{1+\beta}{2} \times \beta \times 2^{\frac{\beta-1}{2}}\right)}\right)^{\frac{1}{\beta}}, \\
X_{i, G+1}=\left\{\begin{array}{c}
X_{i, G 1}, \text { Fitness }\left(X_{i, G 1}\right)<\operatorname{Fitness}\left(X_{i, G}\right), \\
X_{i, G}, \text { Fitness }\left(X_{i, G 1}\right) \geq \operatorname{Fitness}\left(X_{i, G}\right)
\end{array}\right.
\end{gathered}
$$

where $X_{i, G 1}$ represents the solution of the current generation after Lévy flight, $\beta$ is a constant number with a value set to $1.5, \mu$ and $v$ are random numbers obeying the normal distribution, and $\Gamma$ is the gamma function. The Lévy flight strategy improves the local search capability of the individual.

The IWOA can be built by implementing the above strategies for the WOA, and the framework for the proposed algorithm is shown in Figure 2. 


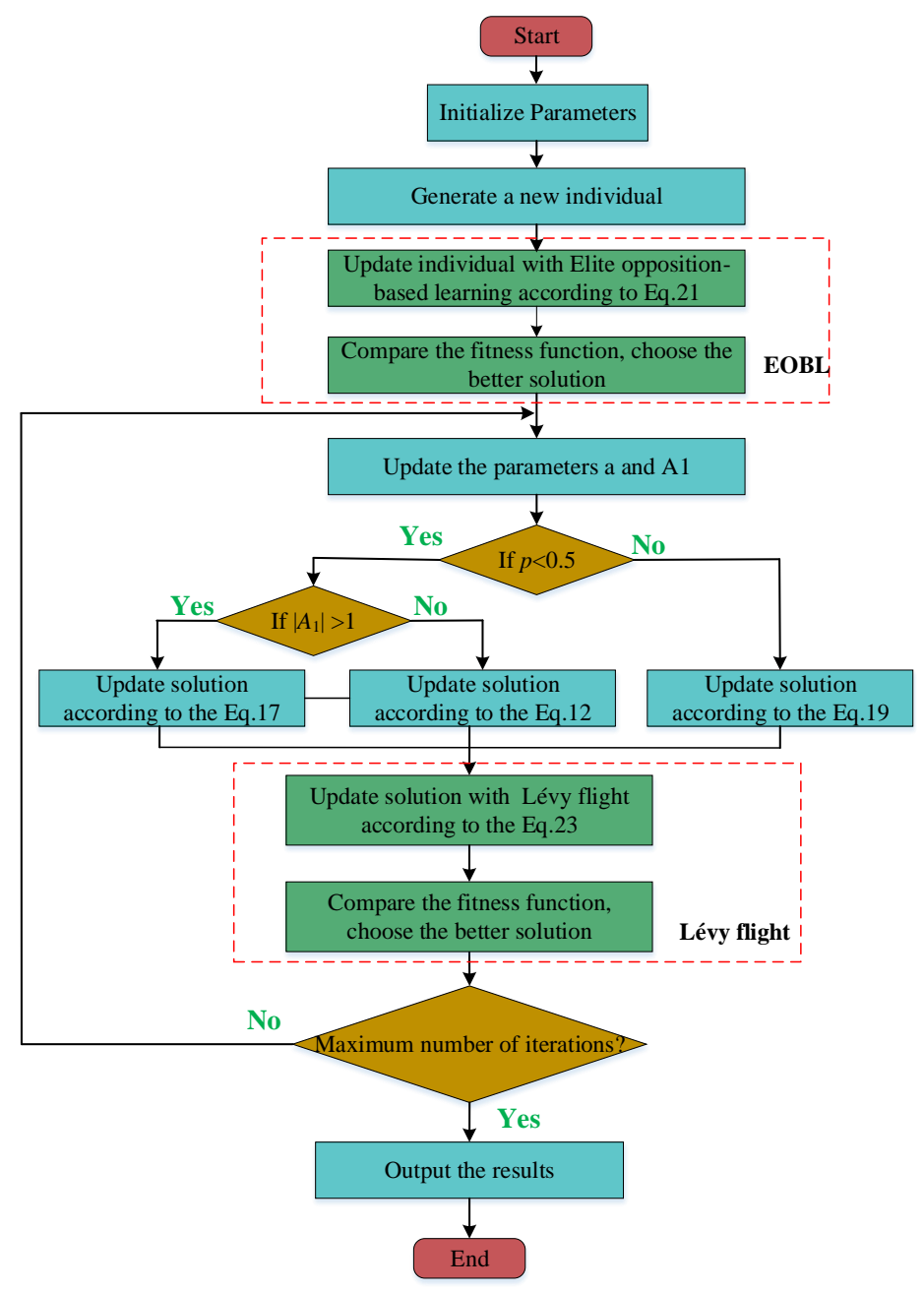

Figure 2. Flowchart of the proposed improved whale optimization algorithm (IWOA).

\section{Simulation Results and Analysis}

Simulations were conducted to validate the performance of the proposed positioning method. Four LEDs were placed on the ceiling of the room as signal generators, with the size of $5 \mathrm{~m} \times 5 \mathrm{~m} \times 6 \mathrm{~m}$, and the coordinates of the LEDs were $[5,0,6]^{T},[0,0,6]^{T}$, $[0,5,6]^{T}$, and $[5,5,6]^{T}$. The encoding information based on code division multiple access (CDMA) modulation is transmitted by each LED, and then this information is received by the PD. The location of the PD can be estimated by the IWOA. The main parameters of the simulation are shown in Table 1.

Table 1. Main parameters of simulation experiment.

\begin{tabular}{cc}
\hline Parameter & Value \\
\hline Emitting power of LED, $P_{t}$ & $2.2 \mathrm{~W}$ \\
Area of PD, $A$ & $1 \mathrm{~cm}^{2}$ \\
Half power angle of LED, $\phi_{1 / 2}$ & $60^{\circ}$ \\
Field of view of PD, $\psi_{c}$ & $90^{\circ}$ \\
Optical concentrator's refractive index, $n$ & 1.5 \\
PD's optical conversion efficiency, $R_{p}$ & $0.5 \mathrm{~A} / \mathrm{W}$ \\
Signal-to-noise ratio, SNR & $45 \mathrm{~dB}$ \\
Population size, $N P$ & 50 \\
Max iteration number, $T_{\max }$ & 100 \\
Logarithmic spiral constant, $b$ & 1 \\
\hline
\end{tabular}


In the actual positioning system based on visible-light communication, the PD has a certain rotation angle when moving. The rotation angle of PD along the $X$-axis is small, the rotation angle along the $Y$-axis is small, and the rotation angle along the $Z$-axis is large. Thus, in order to assess the effectiveness of the proposed location method, simulation analysis was carried out without and with PD rotation.

\subsection{Single-Point Analysis without PD Rotation}

To prove the effectiveness of the IWOA, a single point $(2.0,2.0,2.0)(\mathrm{m})$ was chosen for the simulation research. As shown in Figure 3, the red point is the test point, and the blue points are the individuals in the IWOA. At the initial moment, 50 individuals were randomly distributed in a space of $5 \mathrm{~m} \times 5 \mathrm{~m} \times 6 \mathrm{~m}$, which facilitated the whale population to search for the global optimal solution quickly. With the increasing number of iterations, the scattered individuals moved closer to the test point, and all the individuals converged to the test point at 100 iterations. At this time, the value of the fitness function was $2.7701 \times 10^{-9}$, the positioning error of $2.34 \mathrm{~cm}$ was achieved, and the final estimated location was (2.0086, $2.0041,2.0214)(\mathrm{m})$, indicating that the IWOA had high positioning precision.

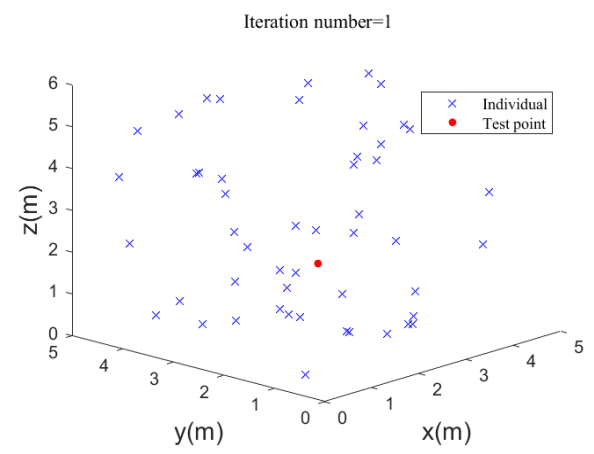

(a)

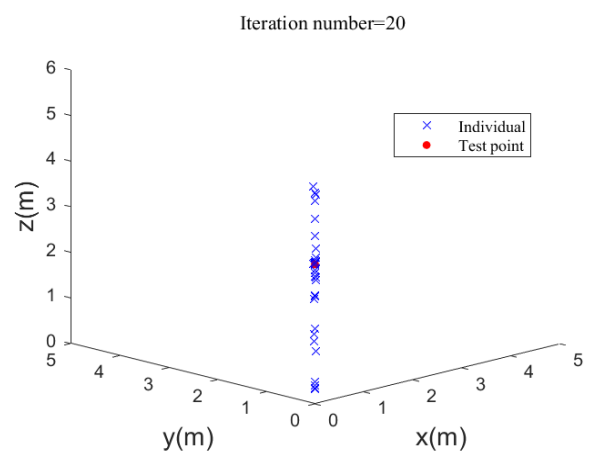

(c)

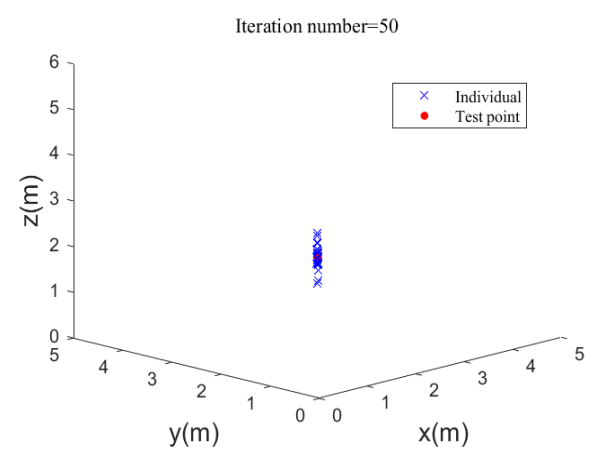

(e)

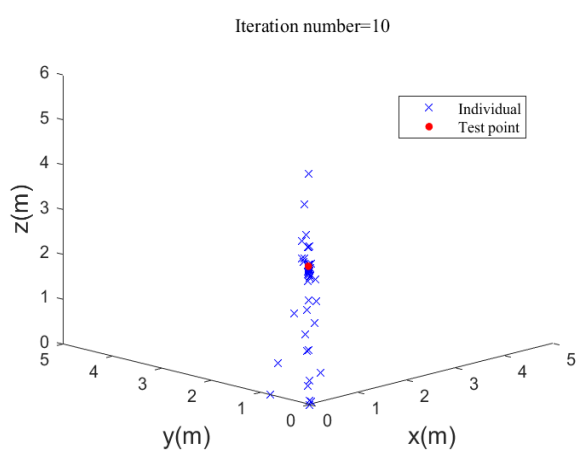

(b)

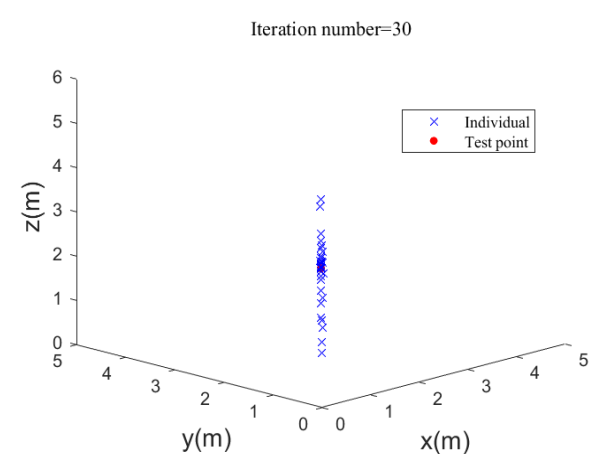

(d)

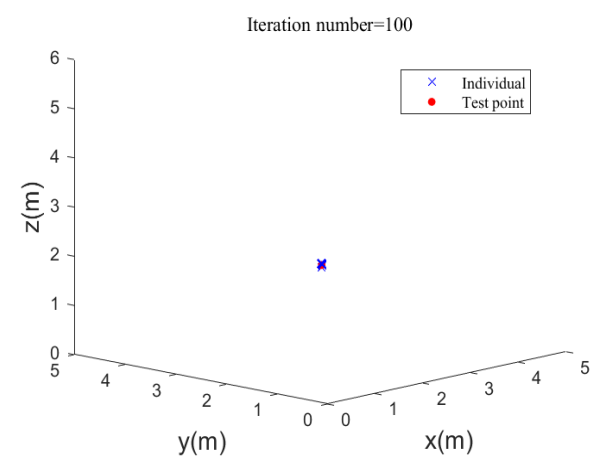

(f)

Figure 3. Convergence process of the improved whale optimization algorithm (IWOA). 
To further demonstrate the effectiveness of the proposed algorithm, IWOA, WOA [27], and MAFSA [18] were simulated in this paper. Simulations were performed for the algorithms under the same conditions. The convergence curves of IWOA, WOA, and MAFSA are shown in Figure 4, indicating that the IWOA improved solution accuracy and was superior to the WOA and MAFSA.

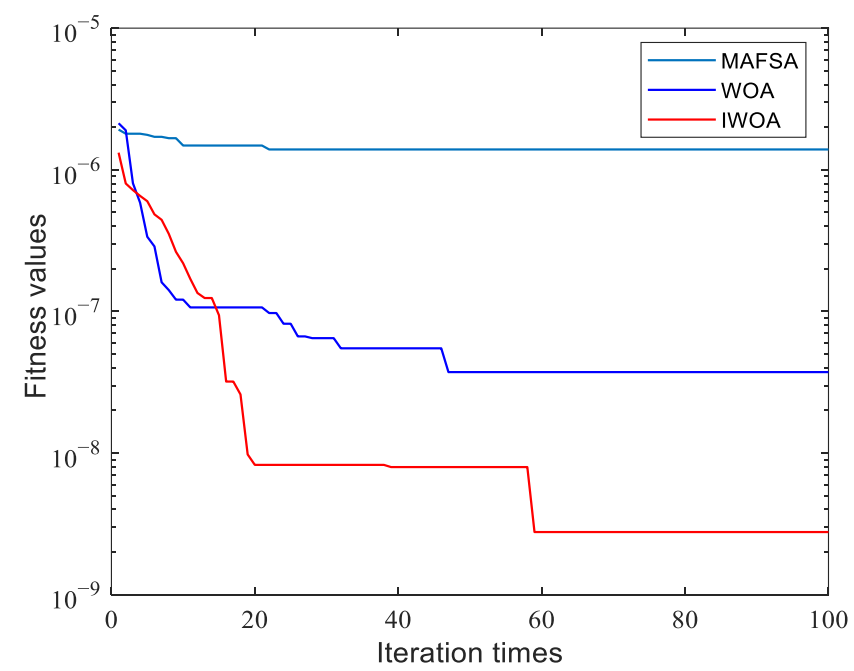

Figure 4. Convergence curves of IWOA, WOA, and MAFSA.

\subsection{Multipoint Analysis without PD Rotation}

To assess the overall positioning performance of the IWOA, the test positions were placed at different heights, i.e., 0.5, 1.0, 1.5, 2.0, 2.5, and $3.0 \mathrm{~m}$, and 2166 test points were generated in the space, the interval between them being $0.25 \mathrm{~m}$ in length and width. To effectively represent the optimization performance of the algorithm, the cumulative distribution function (CDF) of the positioning error was used, and the Euclidean distance was used to express the positioning error.

The PD was considered without any rotation angle for all test points, and the estimated positioning of each test point could be achieved by IWOA optimization. Owing to the randomization of the optimization algorithm searching, the optimization estimation for each test point was run 30 times independently to select the optimal results, and the positioning results at different heights were as shown in Figure 5, where the " $x$ " and " $\triangle$ " symbols represent the real and estimated positions of the test point, respectively. It can be seen from Figure 5a-f that the estimated positions were located close to the real positions, demonstrating the positioning accuracy very well. 

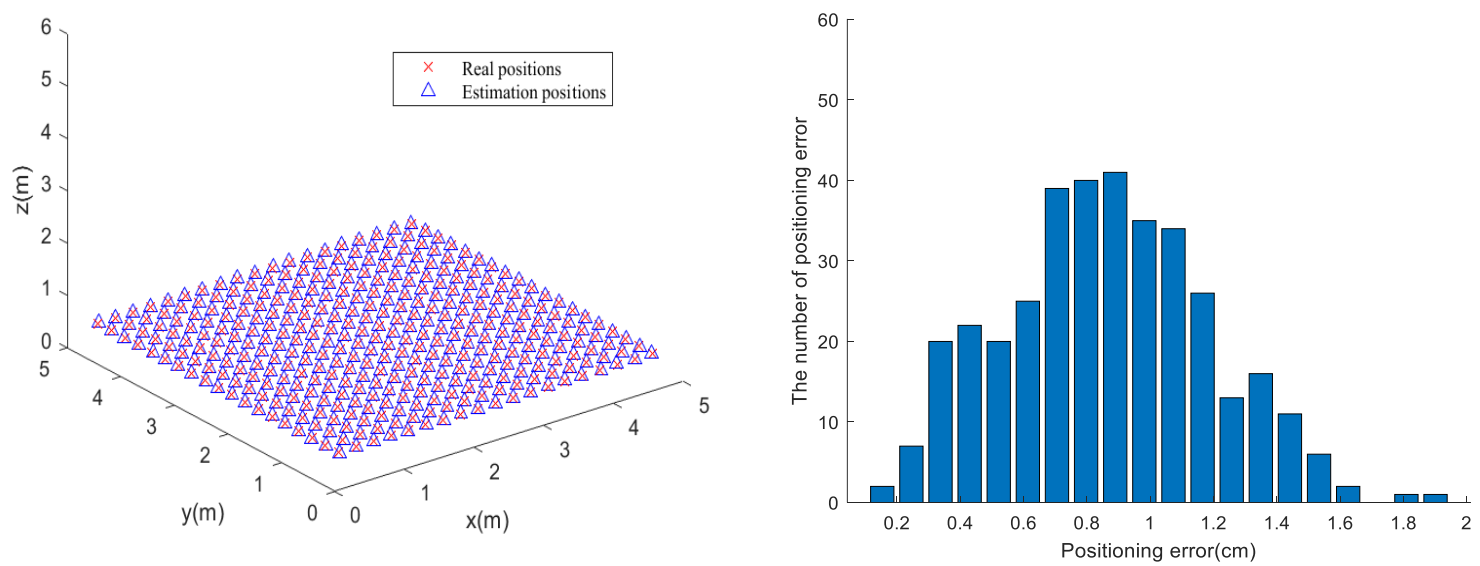

(a)
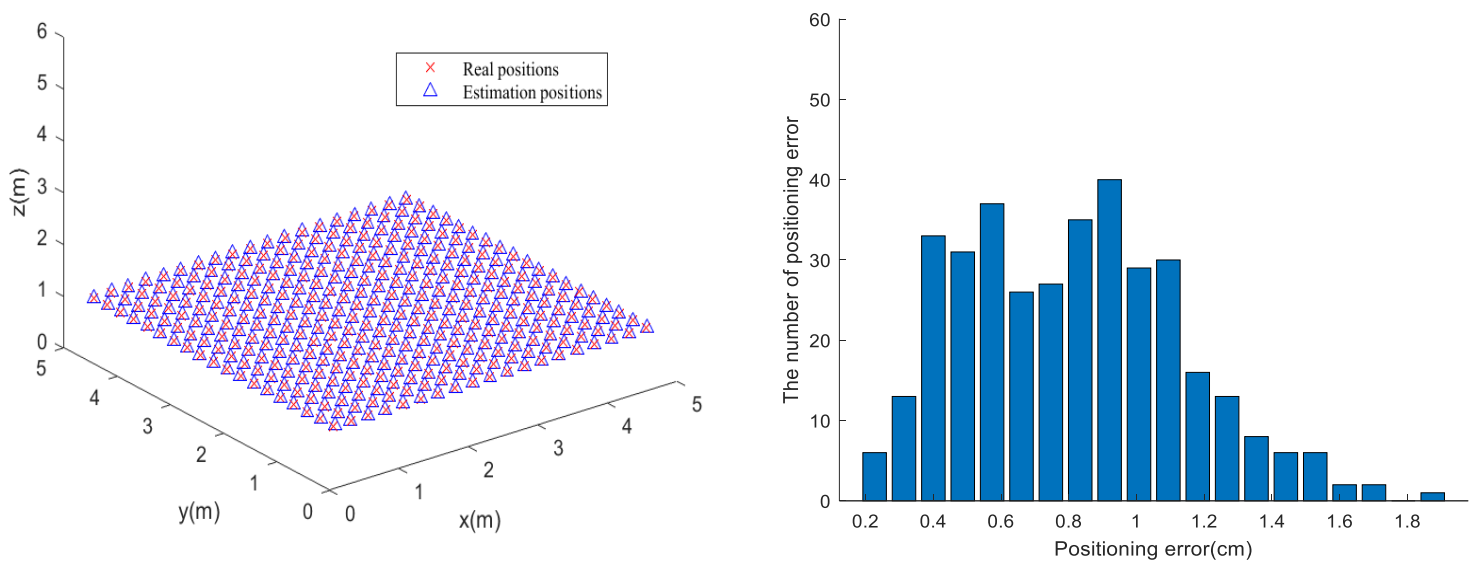

(b)
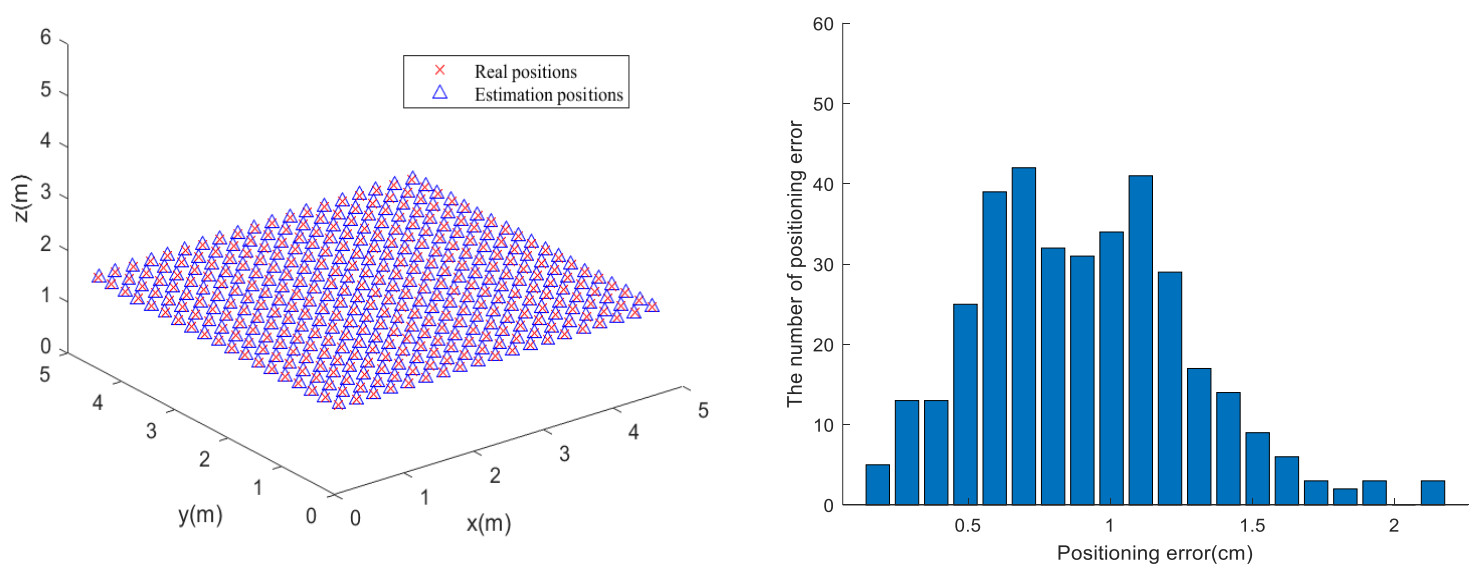

(c)

Figure 5. Cont. 

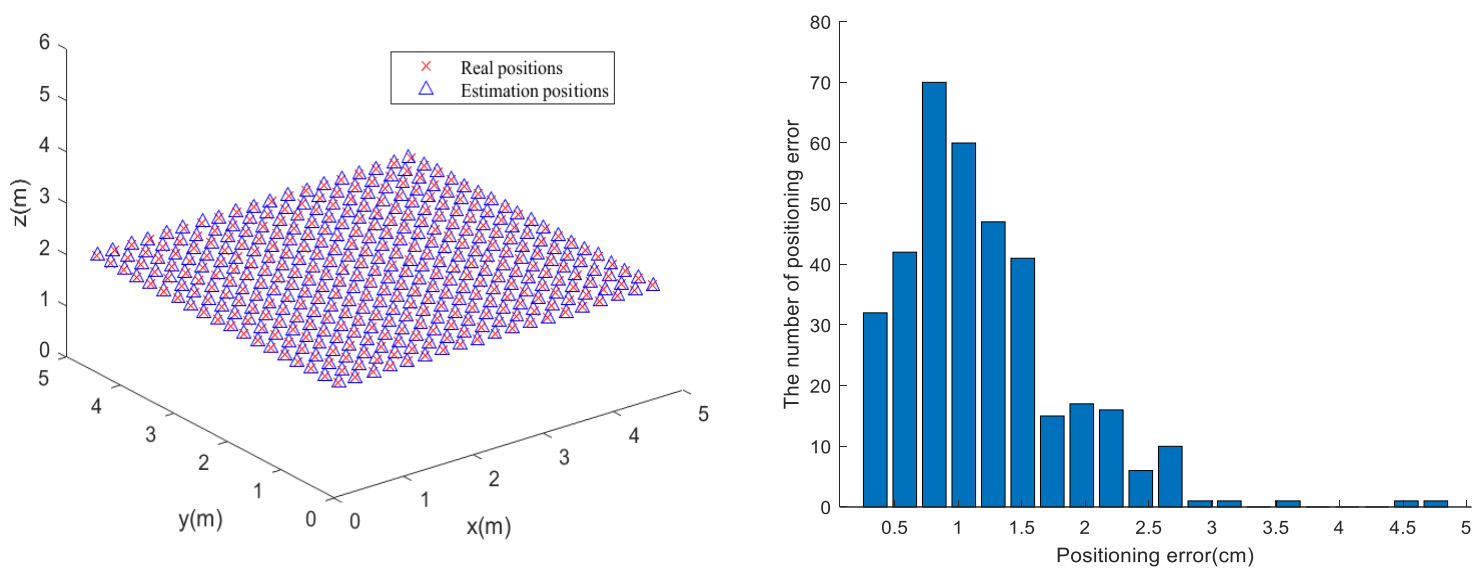

(d)
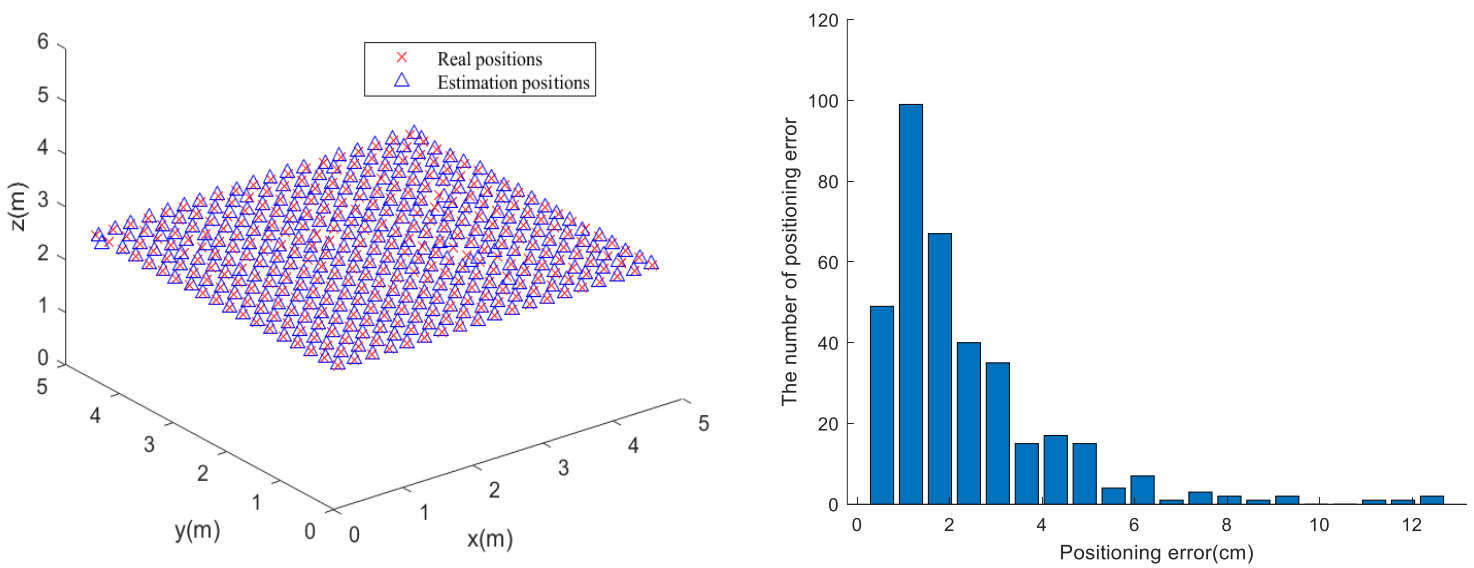

(e)
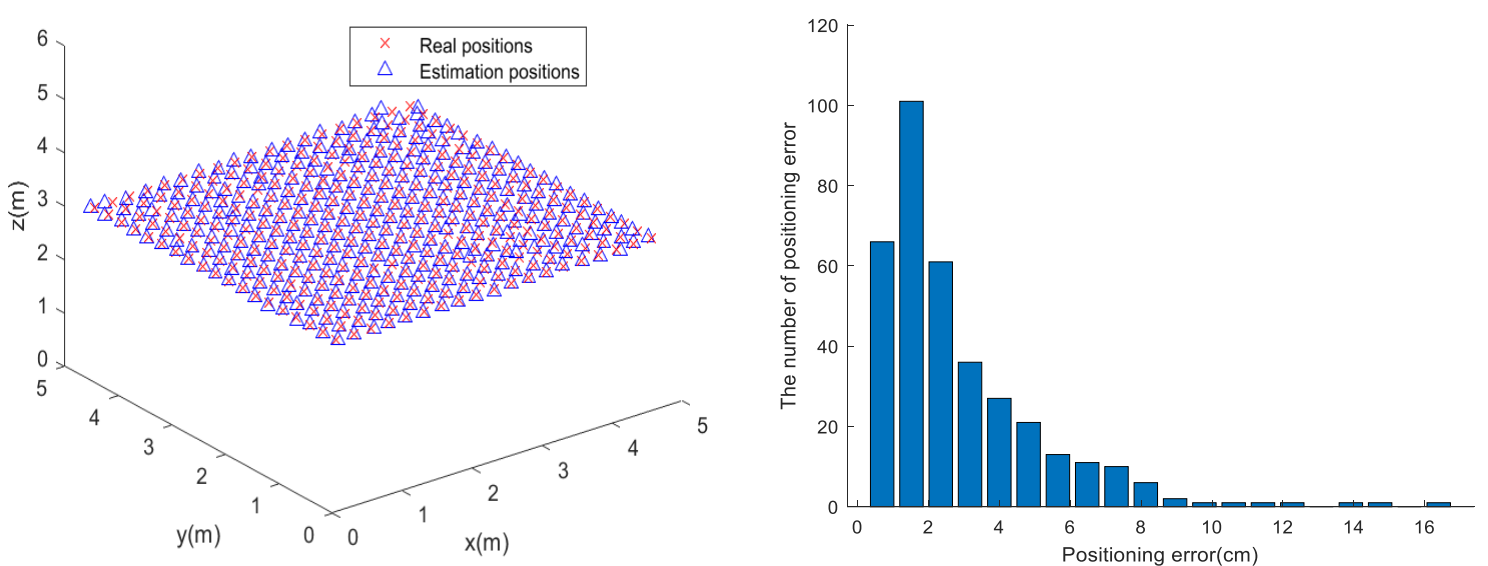

(f)

Figure 5. Real and estimated positions at different heights.

Histograms of the positioning errors with all test points are shown in Figure 6, and the CDF curves are shown in Figure 7. It can be seen from Figure 6 that the average error of $3 \mathrm{D}$ positioning was $2.14 \mathrm{~cm}$, the maximum positioning error was $16.81 \mathrm{~cm}$, and the minimum positioning error was $0.11 \mathrm{~cm}$. As shown in Figure 7, if 95\% of the test points were accepted, the 3D positioning error of the IWOA would be less than $4.49 \mathrm{~cm}$, the horizontal positioning error would be less than $3.05 \mathrm{~cm}$, and the vertical positioning error would be less than $3.78 \mathrm{~cm}$. 


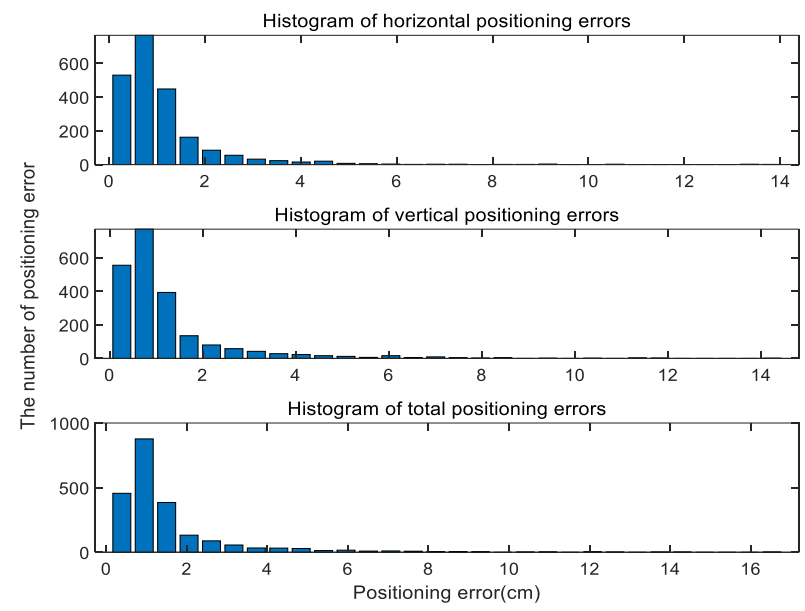

Figure 6. Histograms of total test point positioning errors in three dimensions.

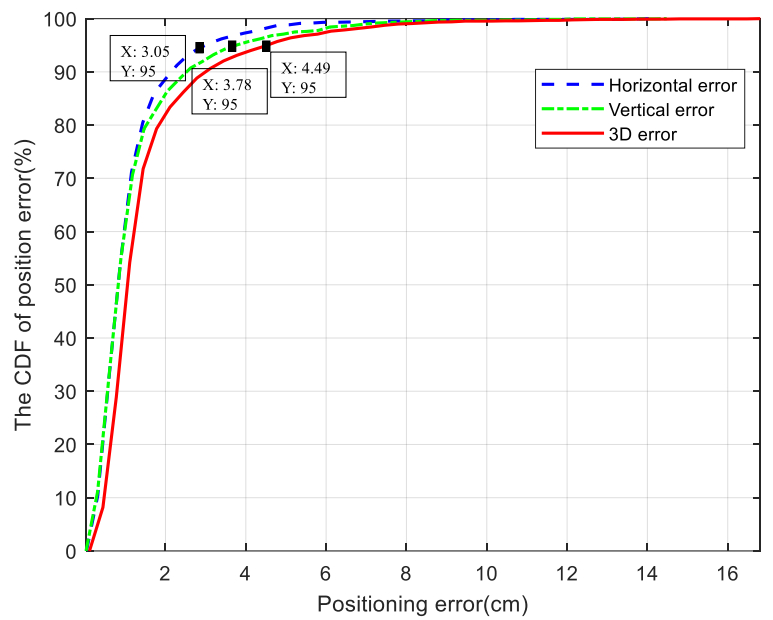

Figure 7. Cumulative distribution function (CDF) curves of total test point positioning errors in three dimensions.

The above experimental results validate that a centimeter level of positioning error can be achieved by the proposed IWOA in an indoor VLP system.

\subsection{Multipoint Analysis with PD Rotation}

In the preceding section, the PD was assumed to have no rotation angle while moving; however, in an actual VLP system, the PD would have a certain rotation angle. To quantify positioning accuracy, it is supposed that rotation angles along the $X$-axis and $Y$-axis are in the ranges $\left[0^{\circ}, 10^{\circ}\right]$ and $\left[0^{\circ}, 10^{\circ}\right]$, and the rotation angle along the $Z$-axis is in the range $\left[0^{\circ}, 360^{\circ}\right]$. The position of all test points was consistent with the preceding section. It was assumed that the PD rotated randomly within the set angle range at each test point. The IWOA was used to estimate the positions of test points when the estimation of angle was ignored and considered. The positioning results when the estimation of rotation angle was ignored are shown in Figure 8, and those when the estimation of rotation angle was considered are shown in Figure 9. 


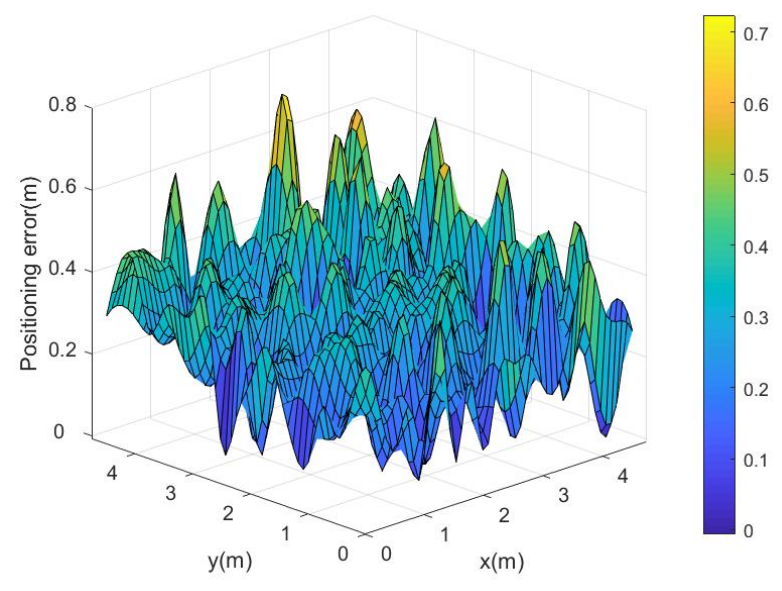

(a) Average positioning error $(z=1.0 \mathrm{~m})$

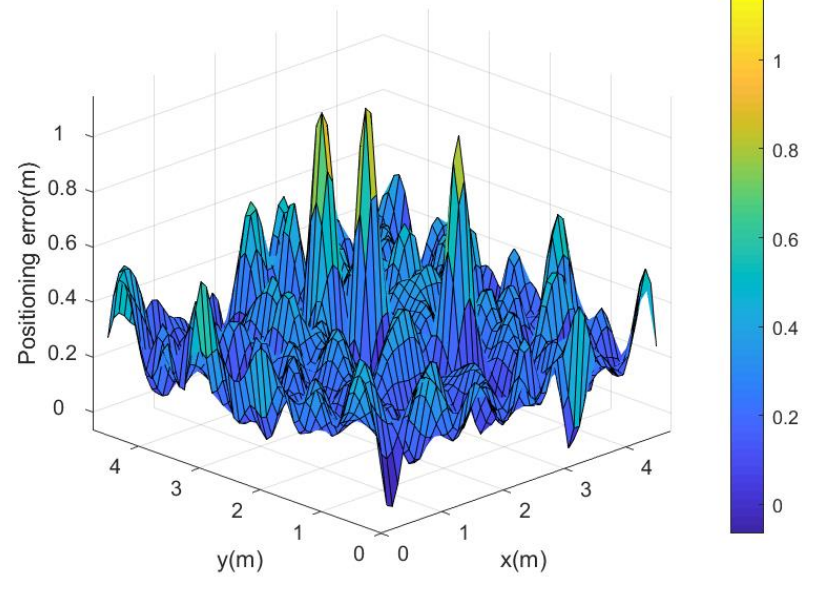

(c) Average positioning error $(z=3.0 \mathrm{~m})$

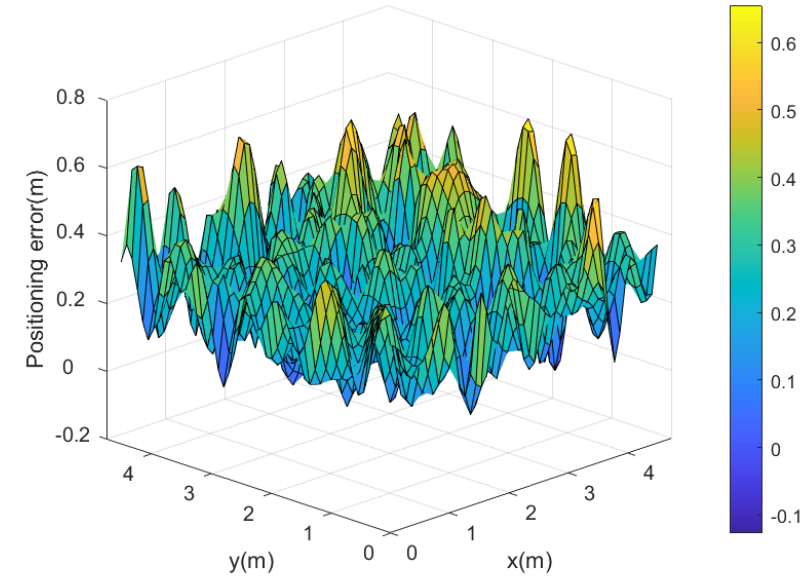

(b) Average positioning error $(z=2.0 \mathrm{~m})$

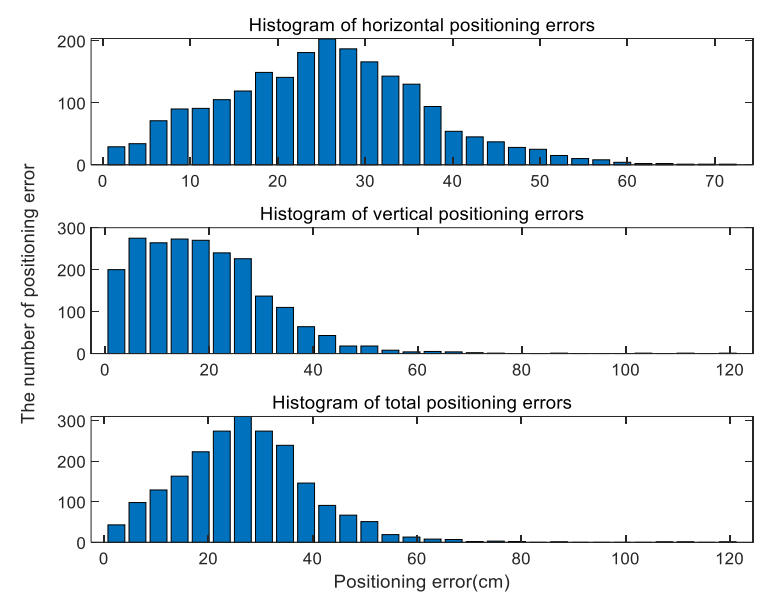

(d) Histograms of positioning error

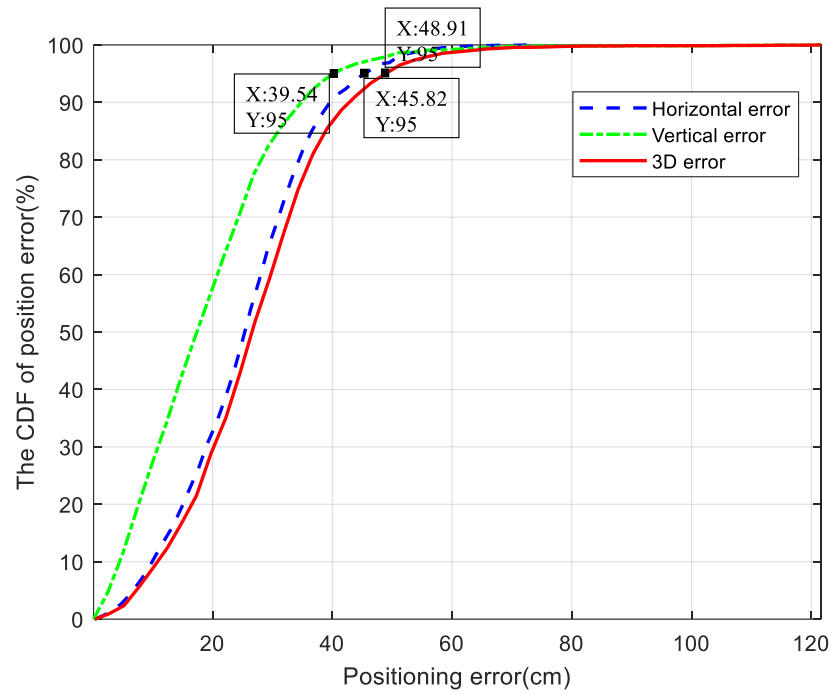

(e) Cumulative distribution function (CDF) curves of positioning error

Figure 8. Results when ignoring angle estimation. 


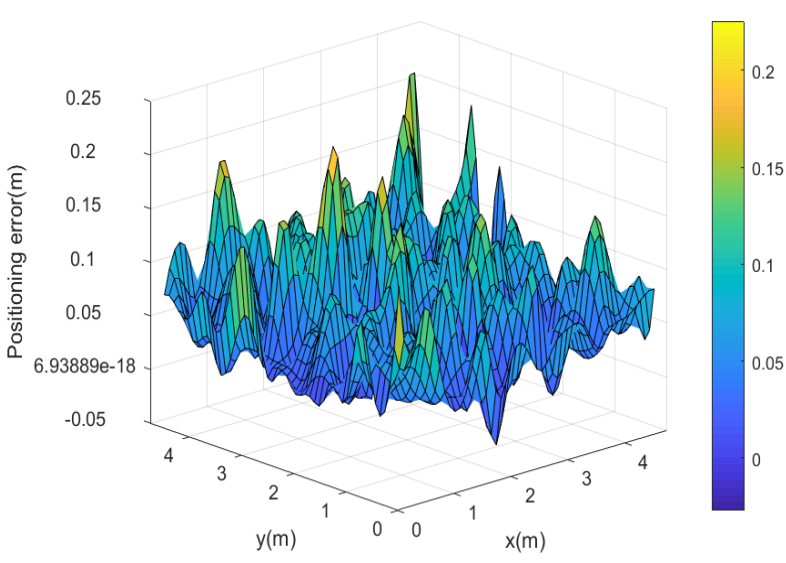

(a) Average positioning error $(z=1.0 \mathrm{~m})$

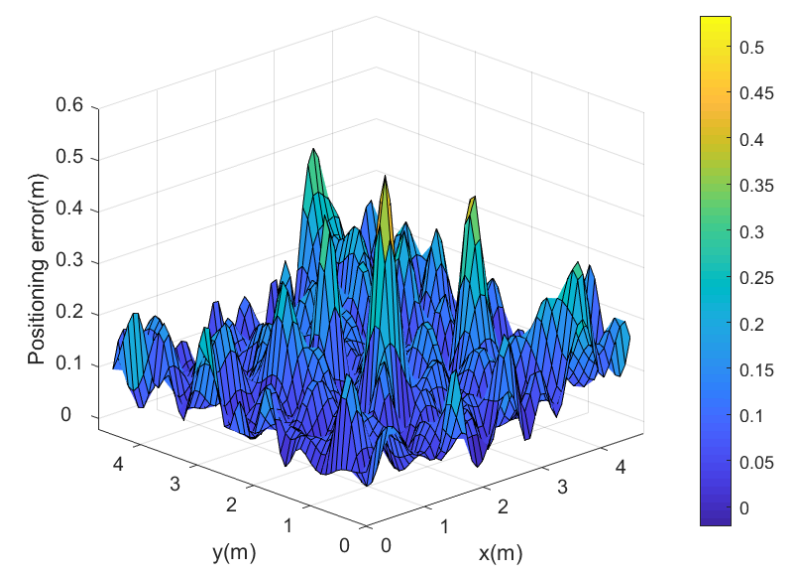

(c) Average positioning error $(z=3.0 \mathrm{~m})$

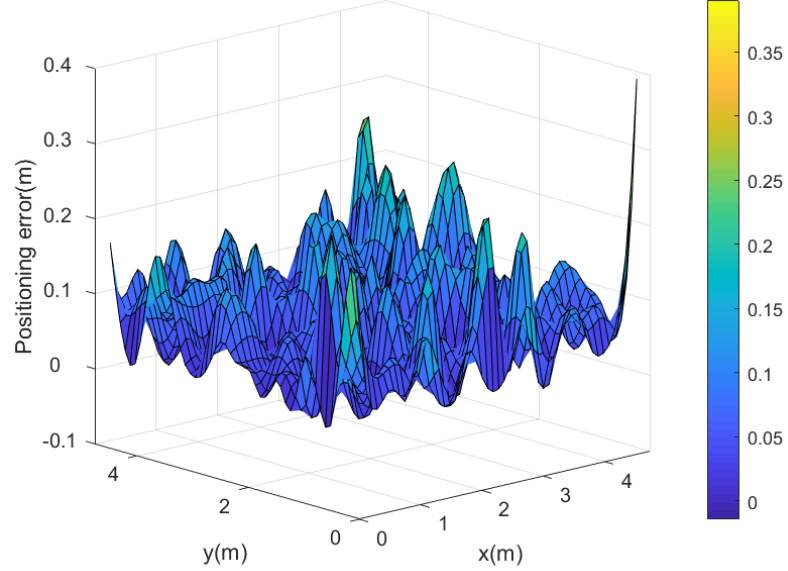

(b) Average positioning error $(z=2.0 \mathrm{~m})$

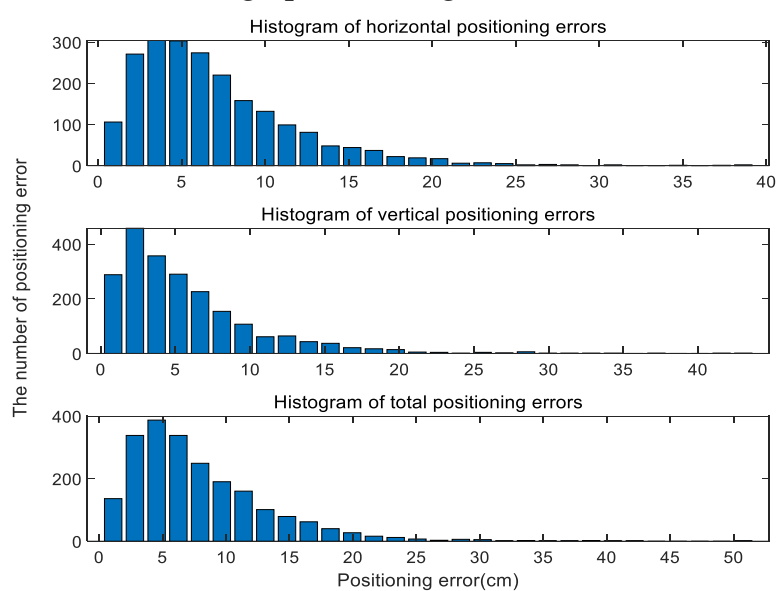

(d) Histograms of positioning error

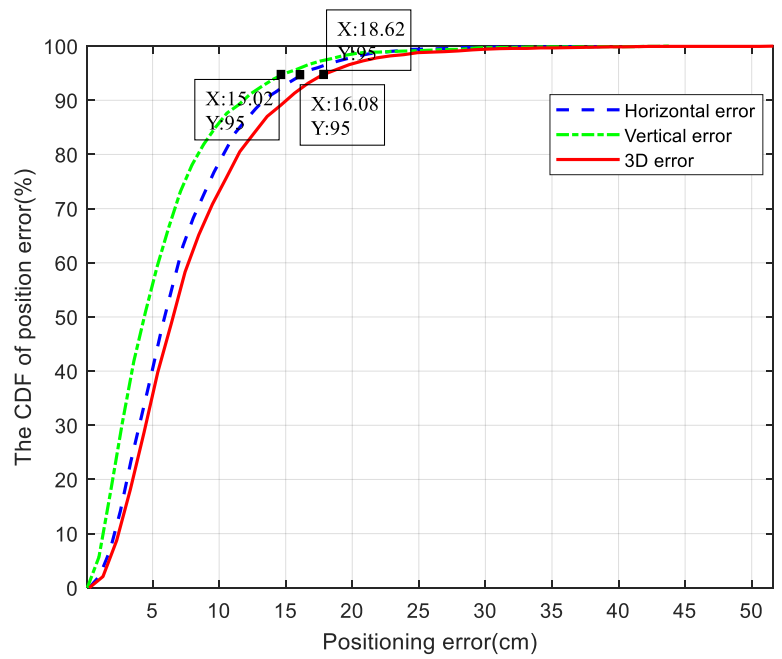

(e) Cumulative distribution function (CDF) curves of positioning error

Figure 9. Results when considering angle estimation.

When the rotation angles along the $X$-axis, $Y$-axis, and $Z$-axis were within the ranges $\left[0^{\circ}, 10^{\circ}\right],\left[0^{\circ}, 10^{\circ}\right]$, and $\left[0^{\circ}, 360^{\circ}\right]$, respectively, the average error of $3 \mathrm{D}$ positioning reached $27.14 \mathrm{~cm}$ when angle estimation was ignored, as shown in Figure $8 \mathrm{a}-\mathrm{e}$. If $95 \%$ of the test points were accepted, a 3D positioning error less than $48.91 \mathrm{~cm}$, a horizontal positioning error less than $45.82 \mathrm{~cm}$, and a vertical positioning error of less than $39.54 \mathrm{~cm}$ would be achieved by the IWOA algorithm. 
When the rotation angles along the $X$-axis, $Y$-axis, and $Z$-axis were within the ranges $\left[0^{\circ}, 10^{\circ}\right],\left[0^{\circ}, 10^{\circ}\right]$, and $\left[0^{\circ}, 360^{\circ}\right]$, respectively, the average error of $3 \mathrm{D}$ positioning was $7.85 \mathrm{~cm}$ when angle estimation was considered, as shown in Figure 9a-e. If 95\% of the test points were accepted, a 3D positioning error less than $18.62 \mathrm{~cm}$, a horizontal positioning error less than $16.08 \mathrm{~cm}$, and a vertical positioning error less than $15.02 \mathrm{~cm}$ would be achieved by the IWOA algorithm. The results show that the IWOA, which took PD rotation angle estimation into account, could significantly improve 3D positioning accuracy.

As explained previously, when the PD was considered without rotation angle, high localization accuracy was achieved by the proposed IWOA positioning optimization algorithm in the VLP system. When the PD was considered with a rotation angle, the positioning simulation results show that the IWOA considering rotation angle estimation outperformed the IWOA ignoring it.

\section{Conclusions}

In this work, a 3D indoor VLP system based on an improved whale optimization algorithm (IWOA) was proposed to reduce the error caused by photodiode (PD) rotation. In addition to introducing the VLC system with the PD rotation angles, a novel IWOA with an elite opposition-based learning strategy and Lévy flight strategy was proposed. The 3D indoor positioning problem with PD rotation was effectively solved by the IWOA. Simulation results show that the average error of 3D positioning was $2.14 \mathrm{~cm}$ without PD rotation angle, and positioning errors less than $4.49 \mathrm{~cm}$ were achieved for $95 \%$ of the test points in 3D positioning. When PD was considered with a rotation angle, the average error of 3D positioning was $27.14 \mathrm{~cm}$, and positioning errors less than $48.91 \mathrm{~cm}$ were achieved in $3 \mathrm{D}$ positioning for $95 \%$ of the test points when ignoring angle estimation. The average error of 3D positioning was $7.85 \mathrm{~cm}$, and positioning errors less than $18.62 \mathrm{~cm}$ were achieved in $3 \mathrm{D}$ positioning for $95 \%$ of test points when the estimation of angle was considered. The proposed method effectively reduced the significant errors resulting from PD rotation in an indoor VLP system, and it can be applied in a variety of indoor location scenes.

Author Contributions: Conceptualization: X.M. and F.H.; methodology: X.M. and C.J.; software: X.M. and C.C.; validation: X.M.; investigation: C.J.; project administration: X.M. and Q.W. All authors have read and agreed to the published version of the manuscript.

Funding: This research was funded by the National Natural Science Fund Projects of China (Grant No. 61702375), the Anhui University Provincial Natural Science Research Project of China (Grant Nos. KJ2019A0869, KJ2020A0785, and KJ2021A1164), the Anhui Province University Quality Engineering Project of China (Grant Nos.2018ylzy073 and 2020jyxm2146), and the Natural Science Key Scientific Research Project of West Anhui University (Grant No. 0041021003).

Institutional Review Board Statement: Not applicable.

Informed Consent Statement: Not applicable.

Data Availability Statement: Not applicable.

Conflicts of Interest: The authors declare no conflict of interest.

\section{References}

1. Khan, L.U. Visible light communication: Applications, architecture, standardization and research challenges. Digit. Commun. Netw. 2017, 3, 78-88. [CrossRef]

2. Siddique, I.; Awan, M.Z.; Khan, M.Y.; Mazhar, A. Li-Fi the Next Generation of Wireless Communication through Visible Light Communication (VLC) Technology. Int. J. Sci. Res. Comput. Sci. Eng. Inf. Technol. 2019, 5, 30-37. [CrossRef]

3. Fang, J.; Yang, Z.; Long, S.; Wu, Z.; Zhao, X.; Liang, F.; Jiang, Z.L.; Chen, Z. High-Speed Indoor Navigation System based on Visible Light and Mobile Phone. IEEE Photonics J. 2017, 9, 8200711. [CrossRef]

4. Huynh, P.; Yoo, M. VLC-Based Positioning System for an Indoor Environment Using an Image Sensor and an Accelerometer Sensor. Sensors 2016, 16, 783. [CrossRef]

5. Davidson, P.; Piche, R. A Survey of Selected Indoor Positioning Methods for Smartphones. IEEE Commun. Surv. Tutor. 2017, 19, 1347-1370. [CrossRef] 
6. Steendam, H.; Wang, T.Q.; Armstrong, J. Theoretical Lower Bound for Indoor Visible Light Positioning Using Received Signal Strength Measurements and an Aperture-Based Receiver. J. Lightwave Technol. 2017, 35, 309-319. [CrossRef]

7. Keskin, M.F.; Gezici, S.; Arikan, O. Direct and Two-Step Positioning in Visible Light Systems. IEEE Trans. Commun. 2018, 66, 239-254. [CrossRef]

8. Wu, Y.; Liu, X.; Guan, W.; Chen, B.; Chen, X.; Xie, C. High-speed 3D indoor localization system based on visible light communication using differential evolution algorithm. Opt. Commun. 2018, 424, 177-189. [CrossRef]

9. Gonendik, E.; Gezici, S. Fundamental Limits on RSS Based Range Estimation in Visible Light Positioning Systems. IEEE Commun. Lett. 2015, 19, 2138-2141. [CrossRef]

10. Du, P.; Zhang, S.; Chen, C.; Yang, H.; Zhong, W.-D.; Zhang, R.; Alphones, A.; Yang, Y. Experimental Demonstration of 3D Visible Light Positioning Using Received Signal Strength with Low-Complexity Trilateration Assisted by Deep Learning Technique. IEEE Access 2019, 7, 93986-93997. [CrossRef]

11. Chaudhary, N.; Alves, L.N.; Ghassemlooy, Z. Impact of Transmitter Positioning and Orientation Uncertainty on RSS-Based Visible Light Positioning Accuracy. Sensors 2021, 21, 3044. [CrossRef] [PubMed]

12. Sun, X.; Hu, C.; Lei, G.; Guo, Y.; Zhu, J. State Feedback Control for a PM Hub Motor Based on Gray Wolf Optimization Algorithm. IEEE Trans. Power Electron. 2020, 35, 1136-1146. [CrossRef]

13. İnci, M.; Caliskan, A. Performance enhancement of energy extraction capability for fuel cell implementations with improved Cuckoo search algorithm. Int. J. Hydrog. Energy 2020, 45, 11309-11320. [CrossRef]

14. Kardani, N.; Bardhan, A.; Roy, B.; Samui, P.; Nazem, M.; Armaghani, D.J.; Zhou, A. A novel improved Harris Hawks optimization algorithm coupled with ELM for predicting permeability of tight carbonates. Eng. Comput. 2021. [CrossRef]

15. Alsalibi, B.; Abualigah, L.; Khader, A.T. A novel bat algorithm with dynamic membrane structure for optimization problems. Appl. Intell. 2020, 51, 1992-2017. [CrossRef]

16. Cai, Y.; Guan, W.; Wu, Y.; Xie, C.; Chen, Y.; Fang, L. Indoor High Precision Three-Dimensional Positioning System Based on Visible Light Communication Using Particle Swarm Optimization. IEEE Photonics J. 2017, 9, 7908120. [CrossRef]

17. Guan, W.; Wu, Y.; Xie, C.; Chen, H.; Cai, Y.; Chen, Y. High-precision approach to localization scheme of visible light communication based on artificial neural networks and modified genetic algorithms. Opt. Eng. 2017, 56, 106103. [CrossRef]

18. Huang, M.; Chen, B.; Jiang, J.; Guan, W.; Cai, X.; Wen, S. High-precision indoor three-dimensional positioning system based on visible light communication using modified artificial fish swarm algorithm. Opt. Eng. 2018, 57, 106102.

19. Xu, S.; Wu, Y.; Wang, X.; Wei, F. Indoor 3D visible light positioning system based on adaptive parameter particle swarm optimisation. IET Commun. 2020, 14, 3707-3714. [CrossRef]

20. Chen, Y.; Zheng, H.; Liu, H.; Han, Z.; Ren, Z. Indoor High Precision Three-Dimensional Positioning System Based on Visible Light Communication Using Improved Hybrid Bat Algorithm. IEEE Photonics J. 2020, 12, 6802513. [CrossRef]

21. Chen, Y.; Ren, Z.; Han, Z.; Liu, H.; Shen, Q.-X.; Wu, Z. LED based high accuracy indoor visible light positioning algorithm. Optik 2021, 243, 166853. [CrossRef]

22. Mathias, L.C.; De Melo, L.F.; Abrao, T. 3-D Localization With Multiple LEDs Lamps in OFDM-VLC System. IEEE Access 2019, 7 , 6249-6261. [CrossRef]

23. Kim, H.-S.; Kim, D.-R.; Yang, S.-H.; Son, Y.-H.; Han, S.-K. An Indoor Visible Light Communication Positioning System Using a RF Carrier Allocation Technique. J. Lightwave Technol. 2013, 31, 134-144. [CrossRef]

24. Chen, H.; Guan, W.; Li, S.; Wu, Y. Indoor high precision three-dimensional positioning system based on visible light communication using modified genetic algorithm. Opt. Commun. 2018, 413, 103-120. [CrossRef]

25. Guan, W.; Wu, Y.; Wen, S.; Chen, H.; Yang, C.; Chen, Y.; Zhang, Z. A novel three-dimensional indoor positioning algorithm design based on visible light communication. Opt. Commun. 2017, 392, 282-293. [CrossRef]

26. Chaochuan, J.; Ting, Y.; Chuanjiang, W.; Mengli, S. High-Accuracy 3D Indoor Visible Light Positioning Method Based on the Improved Adaptive Cuckoo Search Algorithm. Arab. J. Sci. Eng. 2021. [CrossRef]

27. Mirjalili, S.; Lewis, A. The Whale Optimization Algorithm. Adv. Eng. Softw. 2016, 95, 51-67. [CrossRef]

28. Saidala, R.K.; Devarakonda, N. Improved Whale Optimization Algorithm Case Study: Clinical Data of Anaemic Pregnant Woman. In Data Engineering and Intelligent Computing; Springer: Singapore, 2018; pp. 271-281.

29. Sun, Y.; Wang, X.; Chen, Y.; Liu, Z. A modified whale optimization algorithm for large-scale global optimization problems. Expert Syst. Appl. 2018, 114, 563-577. [CrossRef]

30. Mostafa Bozorgi, S.; Yazdani, S. IWOA: An improved whale optimization algorithm for optimization problems. J. Comput. Des. Eng. 2019, 6, 243-259. [CrossRef]

31. Yildiz, B.S.; Pholdee, N.; Bureerat, S.; Yildiz, A.R.; Sait, S.M. Enhanced grasshopper optimization algorithm using elite oppositionbased learning for solving real-world engineering problems. Eng. Comput. 2021. [CrossRef]

32. Zhou, Y.; Wang, R.; Luo, Q. Elite opposition-based flower pollination algorithm. Neurocomputing 2016, 188, 294-310. [CrossRef]

33. Wang, G.; Zhao, Y.; Yuan, Y. A novel heat transfer search algorithm and its application in solar cells. AIP Adv. 2021, 11, 085011. [CrossRef]

34. Iacca, G.; dos Santos, V.C., Jr.; Veloso de Melo, V. An improved Jaya optimization algorithm with Lévy flight. Expert Syst. Appl. 2021, 165, 113902. [CrossRef] 
35. Haklı, H.; Uğuz, H. A novel particle swarm optimization algorithm with Levy flight. Appl. Soft Comput. 2014, 23, 333-345. [CrossRef]

36. Deotti, L.M.P.; Pereira, J.L.R.; Silva, I.C.D., Jr. Parameter extraction of photovoltaic models using an enhanced Lévy flight bat algorithm. Energy Convers. Manag. 2020, 221, 113114. [CrossRef] 